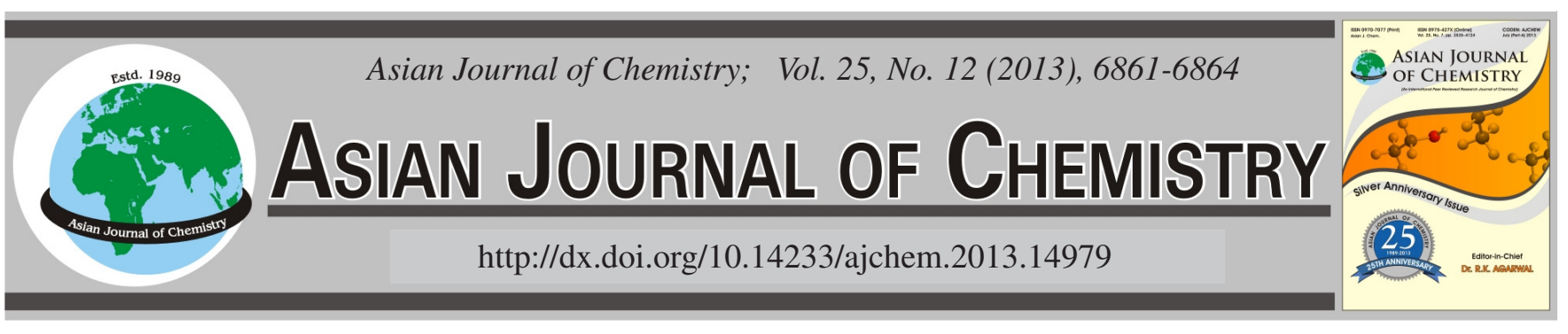

\title{
Mechanical Properties and Morphology of Hyperbranched Polyether/HTPB-PU IPN
}

\author{
XUEJING Song ${ }^{1, *}$ and Yunjun LuO ${ }^{2}$
}

${ }^{1}$ City College of Dongguan University of Technology, Dongguan 523106, P.R. China

${ }^{2}$ School of Material Science and Engineer, Beijing Institute of Technology, Beijing 100081, P.R. China

*Corresponding author: Fax: +86 769 2262527, Tel: +86 13480027882; E-mail: sxj_jing113@163.com

Key Words: Polyurethane, Hyperbranched polyether, Morphology, Mechanical properties, Interpenetrating Polymer Network.

\section{INTRODUCTION}

Hyperbranched polyether has highly-branched and spherical molecular structure. Highly branching structure determines that molecules has no tangles and can not be crystallized; therefore, it can not be used for structural material due to bad mechanical properties ${ }^{1-3}$. However, hyperbranched polymer has unique physical and mechanical properties such as a large number of reactive end groups, good solubility and low viscosity, which make it widely used in functional agent ${ }^{2-4}$. In terms of polymer blending, hyperbranched polymer can act as rheology modifiers, tougheners for thermosets, processing aids as well as compatibilizer of polymer blend ${ }^{3-5}$.

Interpenetrating polymer networks (hereinafter referred to as IPN) is chemical blending technology. It can be defined as combination of two or more polymer in the form of interpenetrating network, among which at least one polymer is polymerization or crosslinked in the presence of another polymer $^{6,7}$. Interpenetrating polymer networks has many advantages such as distinctive network interpenetrating structure, forced compatibility between two phase, interpenetrating interfaces and synergistic effect ${ }^{6-8}$. Two phases in traditional IPN are interpenetrating networks between linear polymer and cross-linked polymers, or among two different cross-linked polymers $^{6-8}$. Hyperbranched polymer has unique architectur which is between linear polymer and cross-linked polymer, this means that if it is taken as one phase in IPN, a new kind of hyperbranchd interpenetrating polymer networks (short for $\mathrm{H}-$ IPN) will be formed. The research introduces hyperbranched polymer into IPN by in situ polymerization of hydroxylterminated polybutadiene-based polyurethane (HTPB-PU) in the presence of hyperbranched polyether, studies mechanical properties, chemical structure and morphology of H-IPN mentioned above and discusses the relationship between structure and mechanical properties.

\section{EXPERIMENTAL}

2-Ethyl-2-(hydroxymethyl)-1,3-propanediol (TMP), $p$ toluenesulfonic acid ( $p$-TSA), Boron trifluoride diethyl ether $\left(\mathrm{BF}_{3} \cdot \mathrm{OEt}_{2}\right)$ were purchased from Beijing chemical factory; $3-$ methyl-3-oxetanemethanol (HMMO), made in laboratory. Hydroxyl-terminated polybutadiene (HTPB, with the numberaverage molecular weight of $4050 \mathrm{~g} / \mathrm{mol}$ and the hydroxyl value of $0.515 \mathrm{mmol} / \mathrm{g}$ ) was supplied by Li Ming Chemical Academy. Isophorone diisocyanate (IPDI) was purchased from Fluka (Germany). Trithenyl bismuth (TPB) was supplied by Shanghai Organic Graduate School. Potassium bromide (KBr), purchased from Aldrich. Before the last four materials were used, they were dried for $4 \mathrm{~h}$ in a vacuum drier at $80^{\circ} \mathrm{C}$.

General procedure: The pseudo-one-step method was adopted to synthesize hyperbranched polyether to obtain higher degree of branching than of one-step method does. Hydroxylterminated hyperbranched polyether synthesized by ring opening polymerization, which took TMP as a core and HMMO as 
monomer, $\mathrm{BF}_{3} \cdot \mathrm{OEt}_{2}$ catalyst. The resultant hyperbranched polyethers with the generation from 1 to 5 are marked with $\mathrm{P}_{10}, \mathrm{P}_{20}, \mathrm{P}_{30}, \mathrm{P}_{40}, \mathrm{P}_{50}$, respectively. Hyperbranched polyethers with hydroxyl terminated groups exhibit poor miscibility with HTPB. In order to improve thermodynamically miscible of both polymers, stearic acid was served as end-capped modifier of hyperbranched polyether, The resultant stearic acid esterterminated hyperbranched polyester (short for SHPE) with generation 1 to 5 are marked with $\mathrm{P}_{11}, \mathrm{P}_{21}, \mathrm{P}_{31}, \mathrm{P}_{41}, \mathrm{P}_{51}$, respectively. The detailed synthesis procedure was described elsewhere ${ }^{9,10}$.

Stoichiometric amount of SHPE, HTPB, IPDI and catalyst (TPB)were fully mixed in a beaker with vigorously stirring at $60{ }^{\circ} \mathrm{C}$ for about $0.5 \mathrm{~h}$. The mixture was then degassed for another $1 \mathrm{~h}$ at $60{ }^{\circ} \mathrm{C}$. The freshly prepared matrix was cast into Teflon molds and cured for 7 days at $60^{\circ} \mathrm{C}, \mathrm{H}-\mathrm{IPN}$ was formed. Control sample is HTPB-PU, which was prepared by the same way except adding SHPE.

Detection method: Infrared Fourier transform spectrometer (FTIR, Nicolet8700) is conducted to characterize chemical state of samples. Phase morphology of IPN was characterized using transmission electron microscopy (TEM, Hitachi HE-800) at $300 \mathrm{kV}$. Polymer specimens were immersed overnight in a solution of $\mathrm{RuO}_{4}$ using an ultramicrotome (Sweden LKB-5) and a 35 diamond knife (Diatome). The cured samples were tested for their mechanical properties at room temperature and a cross-head speed of $50 \mathrm{~mm} / \mathrm{min}$ using a conventional uniaxial testing system (Hewlett-Packard 6022 type Instron).

\section{RESULTS AND DISCUSSION}

Mechanical properties of samples: Tensile test is conducted for mechanical properties of H-IPN with SHPE of different generation and content. The result is shown in Figs. 1 and 2.

From Fig. 1, it can be seen that the maximum tensile strength of the H-IPN increases first and reaches peak, then decreases with the increasing content of SHPE. When $30 \mathrm{wt} \%$ around SHPE with different generation is added, tensile strength of H-IPN reaches the maximum. At the same time, with the generation increasing of SHPE, tensile strength of

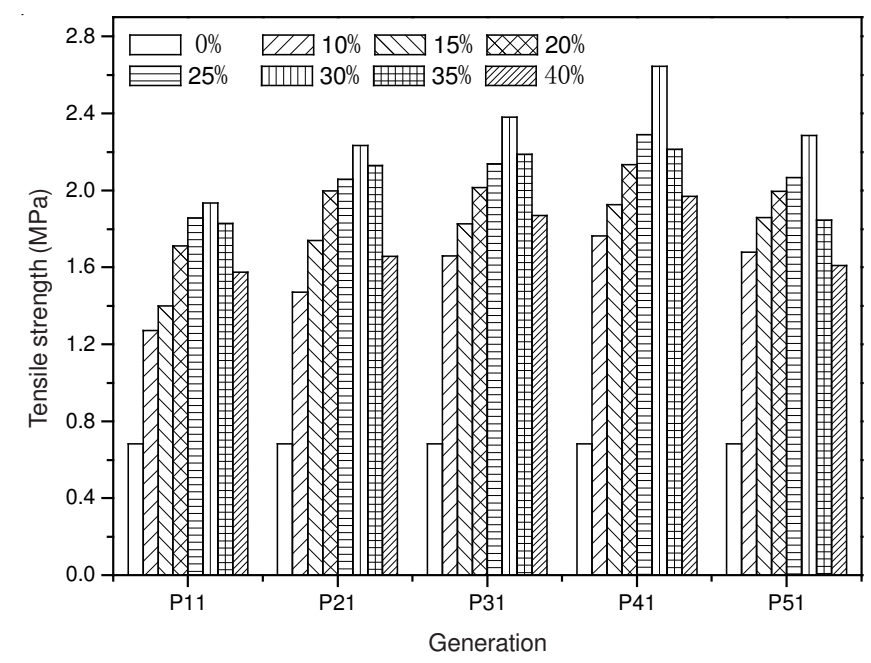

Fig. 1. Effect of composition and generation of SHPE on ultimate tensile strength of H-IPN

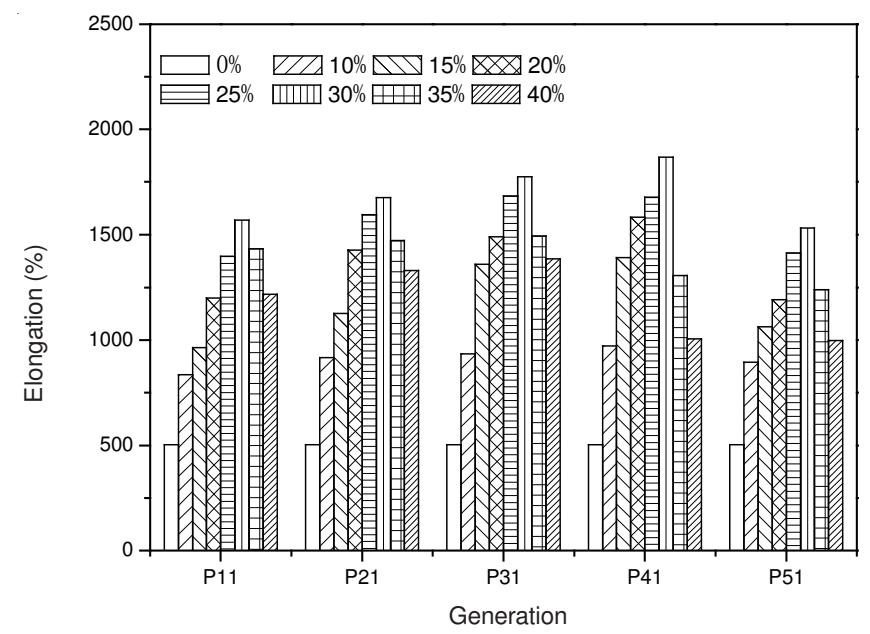

Fig 2. Effect of composition and generation of SHPE on elongation of H-IPN

IPN system reaches peak that $\mathrm{P}_{41}$ is added. When $\mathrm{P}_{41}$ in $\mathrm{P}_{41} /$ HTPB-PU system reaches $30 \mathrm{wt} \%$, the maximum tensile strength of IPN amounts to $2.65 \mathrm{MPa}, 3.86$ times than that of neat HPTB-PU. Variation tendency of elongation (Fig. 2) with the changes of generations and contents of SHPE is similar to that of tensile strength. When $\mathrm{P}_{41}$ in $\mathrm{P}_{41} / \mathrm{HTPB}-\mathrm{HTPB}-\mathrm{PU}$ system reaches $30 \mathrm{wt} \%$, the maximum elongation of IPN amounts to $1868 \%, 3.7$ times than that of control sample. Tensile strength and elongation reach maximum at the same time, this phenomenon shows obviously synergistic effect of IPN.

Chemical states of samples: Infrared spectra of HTPB, $\mathrm{P}_{41}$, HTPB-PU and $\mathrm{P}_{41} /$ HTPB-PU are displayed in Fig. 3. Comparing IR spectra of HTPB, HTPB-PU and $\mathrm{P}_{41} / \mathrm{HTPB}-\mathrm{PU}$, stretching vibration absorption peak ( $c a .3400 \mathrm{~cm}^{-1}$ ) of hydroxyl (in HPTB) disappears when HTPB-PU is formed. Meanwhile, a new broad peak appears around $1509 \mathrm{~cm}^{-1}$, which responding to the bending vibration of nitrogen-hydrogen bond in urethane. On comparing the infrared absorption peak of $\mathrm{P}_{41}$ to that of $\mathrm{P}_{41} /$ HTPB-PU IPN, we can find that absorption peak of ester group in $\mathrm{P}_{41}$ moves from 1739 to $1731 \mathrm{~cm}^{-1}$, red-shifted $8 \mathrm{~cm}^{-1}$. This phenomenon indicates $\mathrm{H}$-bond is formed between ether bond (in $\mathrm{P}_{41}$ ) and amido group (in HTPB-PU) and thus strengthens interaction force between two networks.

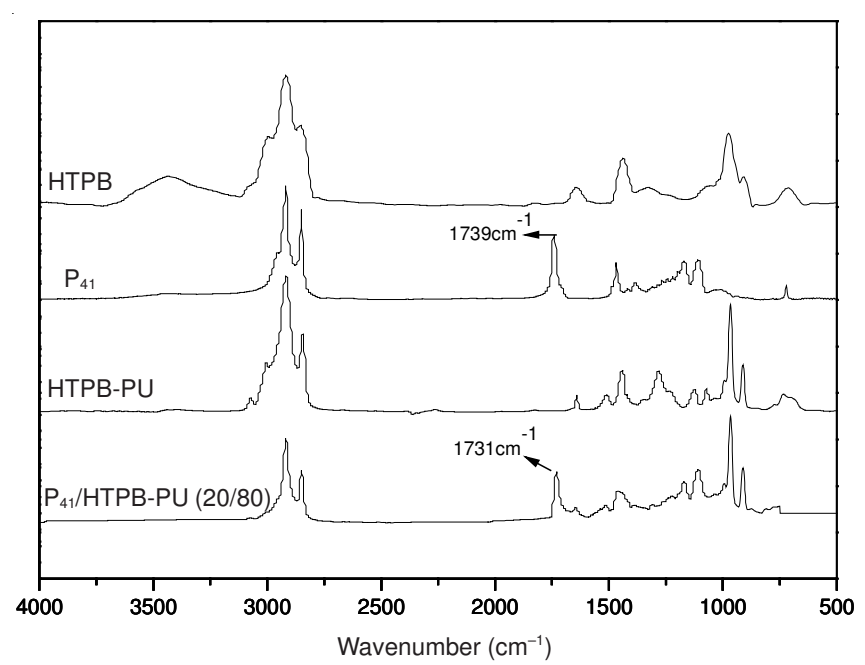

Fig. 3. FTIR spectra of HTPB, $\mathrm{P}_{41}$, HTPB-PU and $\mathrm{P}_{41} / \mathrm{HTPB}-\mathrm{PU}$ 
Morphological properties of samples: As tensile strength and elongation of all H-IPNs reach the peak, when the content of SHPE is $30 \mathrm{wt} \%$ around, no mater which generation. The paper selects H-IPN with content of 30 wt \% SHPE as example,investigating morphology of H-IPN vary with the generation of SHPE changed. Also because mechanical properties of all H-IPNs reach the peak, when $\mathrm{P}_{41}$ is induced into H-IPN. So $\mathrm{P}_{41} / \mathrm{HTPB}-\mathrm{PU}$ with different composition ratios are selected to analyze morphology changed with the content of SHPE. Fig. 4 gives TEM images of H-IPN with 30 wt \% SHPE of different generations. In TEM image of H-IPN (Fig. 4), it is not difficult to observe a dual-phase morphology of $\mathrm{H}-\mathrm{IPN}$, in which the darker areas are HTPB-PU (stained by $\mathrm{OsO}_{4}$ ) and the tinge areas are SHPE. White spherical or oval SHPE is uniformly distributed within HTPB-PU matrix. Interface of the two phases is vague, showing that SHPE is compatible with HTPB-PU. From A to E in Fig. 4, size of dispersed phase gradually increases with the generation increasing of SHPE and the average size is $0.2 \mu \mathrm{m}$ (A: $30 \mathrm{wt} \%$ $\left.\mathrm{P}_{11}\right) ; 0.4 \mu \mathrm{m}\left(\mathrm{B}: 30 \mathrm{wt} \% \mathrm{P}_{21}\right) ; 0.5 \mu \mathrm{m}\left(\mathrm{C}: 30 \mathrm{wt} \% \mathrm{P}_{31}\right) ; 0.8 \mu \mathrm{m}$ (D: 30 wt $\left.\% \mathrm{P}_{41}\right) ; 1.2 \mu \mathrm{m}$ (E: 30 wt $\% \mathrm{P}_{51}$ ), respectively. The SHPE size rises along with the increasing generation. It may have conjunction with molecular weight. Generally speaking, high molecular weight accelerates phase separation ${ }^{11}$. Higher generation of SHPE have higher molecular weight, so the size of SHPE increases as generation rise.

Morphological characterization of H-IPN with the same generation but different content of SHPE is illustrated in Fig. 5. From Fig. 5, it can be seen that when the content of $\mathrm{P}_{41}$ is increased from 10 to $30 \mathrm{wt} \%$, size of dispersed phase enlarged slightly and average size is $0.3 \mu \mathrm{m}\left(\mathrm{A}: 10 \mathrm{wt} \% \mathrm{P}_{41}\right)$; $0.5 \mu \mathrm{m}\left(\mathrm{B}: 20 \mathrm{wt} \% \mathrm{P}_{41}\right) ; 0.8 \mu \mathrm{m}\left(\mathrm{C}: 30 \mathrm{wt} \% \mathrm{P}_{41}\right)$, respectively. When the content of $\mathrm{P}_{41}$ reaches 40 wt \%, H-IPN displays bicontinuous structure. From A to $\mathrm{C}$ of Fig. 5, the size of dispersed phase increasing is caused by the growing proportion of $\mathrm{P}_{41}$ in H-IPN system. In D (Fig. 5), bicontinuous microstructure of H-IPN is related to low viscosity of SHPE. Usually, components of low viscosity in blending tend to form continuous phase ${ }^{12}$.

\section{Conclusion}

The paper studies mechanical properties, molecular structure and morphology of SHPE/HTPB-PU IPN. According to the results of mechanical experiments, it can be concluded

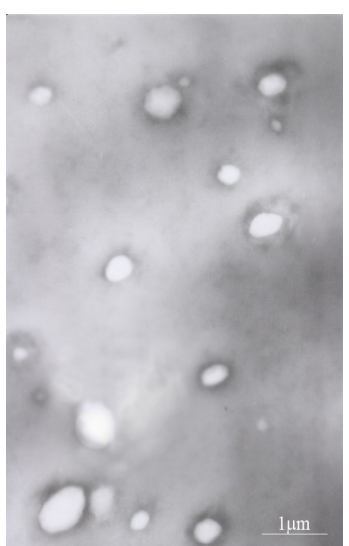

A

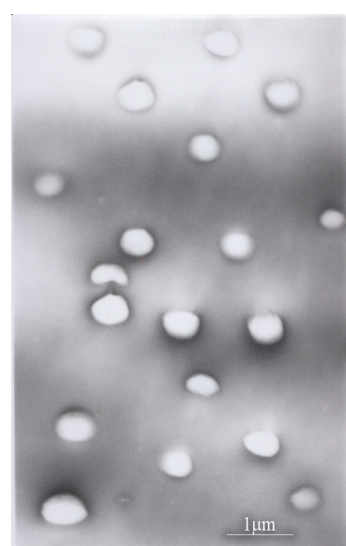

B

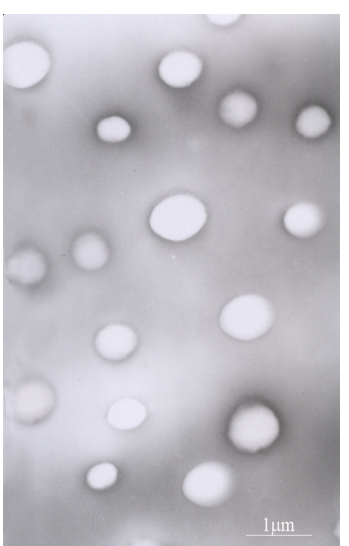

$\mathrm{C}$

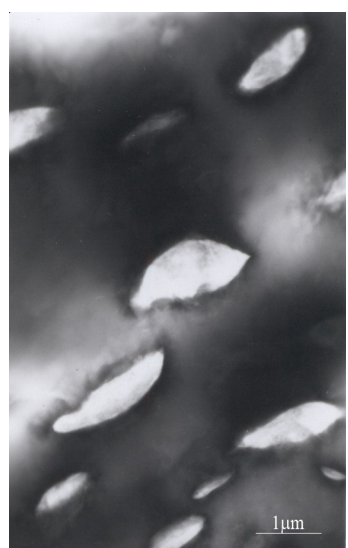

$\mathrm{D}$

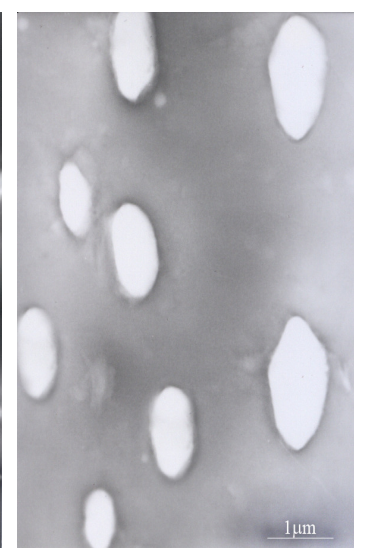

$\mathrm{E}$

Fig. 4. TEM micrographs of 30wt $\%$ different generation SHPE/HTPB-PU IPN (A: $\mathrm{P}_{11} / \mathrm{HTPB}-\mathrm{PU} ; \mathrm{B}: \mathrm{P}_{21} / \mathrm{HTPB}-\mathrm{PU}$; C: $\mathrm{P}_{31} / \mathrm{HTPB}-\mathrm{PU}$; D: $\left.\mathrm{P}_{41} / \mathrm{HTPB}-\mathrm{PU} ; \mathrm{E}: \mathrm{P}_{51} / \mathrm{HTPB}-\mathrm{PU}\right)$

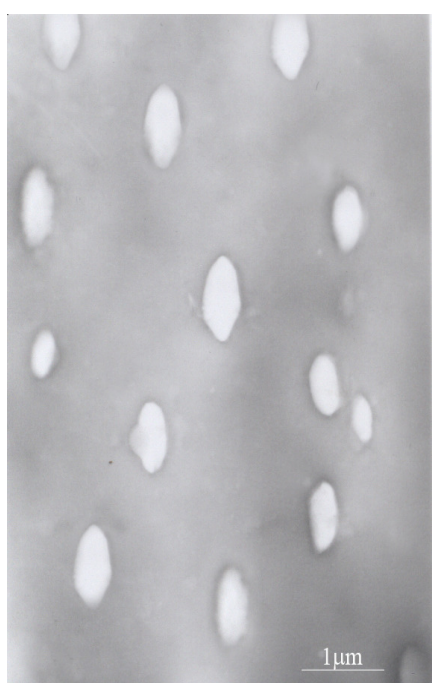

A

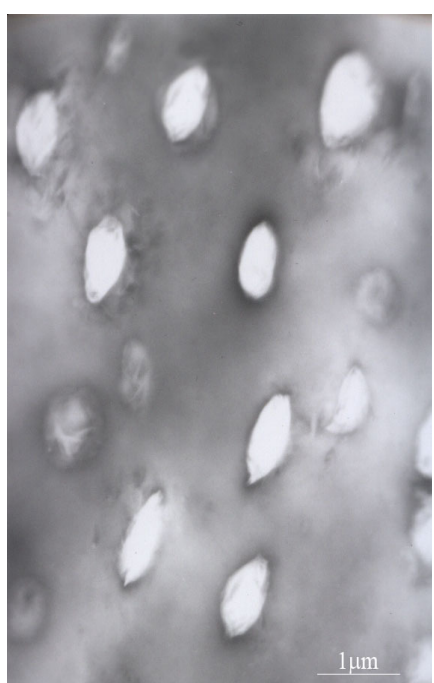

B

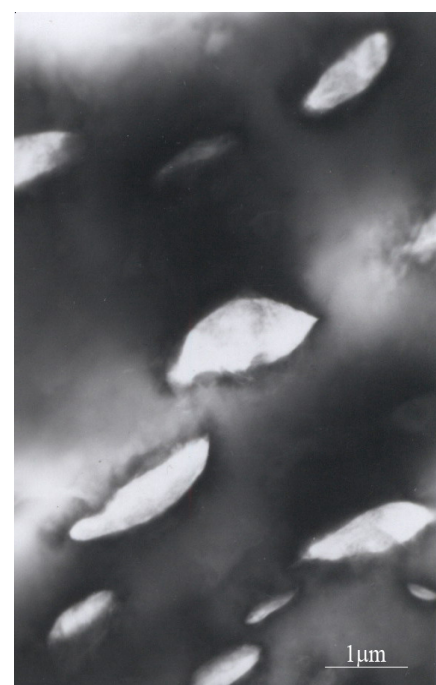

C

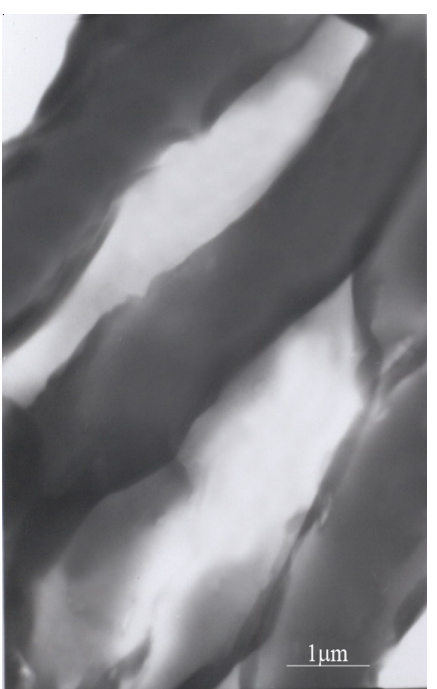

D

Fig. 5. TEM micrographs of $\mathrm{P}_{41} / \mathrm{HTPB}-\mathrm{PU}$ IPN for different content of $\mathrm{P}_{41}$ (A: 1:9; B: 2:8; C: 3:7; D:4:6) 
that the incorporation of SHPE into HTPB-PU exhibits a synergistic effect for H-IPN. Both tensile strength and elongation are enhanced greatly. It may be explained from the following aspects. The one is ascribed to the $\mathrm{H}$-bond interactions developed between two networks as confirmed by the FTIR results. The second may be due to morphology separation in H-IPN which is supported by the TEM analysis. It was also reported in the literature ${ }^{13}$ that the toughness of composites depended on the domain size of secondary phase and always has a maximum value. The optimum domain size could be variable for different resins. For the SHPE/HTPB-PU system, this optimum domain size was about $0.8 \mu \mathrm{m}$. While if SHPE is continuous phase, mechanical properties of H-IPN will be diminished.

\section{ACKNOWLEDGEMENTS}

This work is supported by the Fundamental Researching Fund of Guangdong Provincial Department of Science and Technology (No. 2010B090500004).

\section{REFERENCES}

1. B. Voit, J. Polym. Sci. Part A: Polym. Chem., 43, 2679 (2005).

2. F. Schallausky, M. Erber, H. Komber and A. Lederer, Macromol. Chem. Phys., 209, 2331 (2008).

3. C. Gao and D.Y. Yan, Prog. Polym. Sci., 29, 183 (2004),

4. D. Ratna and G. Simon, J. Appl. Polym. Sci., 117, 557 (2010).

5. D. Foix, X. Ramis, F. Ferrando and A. Serra, Polym. Int., 61, 727 (2012).

6. C. Plesse, F. Vidal, C. Gauthier, J.M. Pelletier, C. Chevrot and D. Teyssiéa, Polymer, 48, 696 (2007).

7. C.M. Paranhos, R.N. Oliveira, B.G. Soares and L.A. Pessan, Macromol. Mater. Res., 10, 43 (2007).

8. Y. Luo, Y. Miao and F. Xu, Appl. Mech. Mater, 66-68, 170 (2011).

9. Y. Konno, H. Kudo, A. Kameyama and T. Nishikubo, J. Polym. Sci., Part A: Polym. Chem., 42, 2543 (2004).

10. L. Ye, Z.G. Feng, Y.M. Zhao, F. Wu, S. Chen and G. Wang, J. Polym. Sci., Part A: Polym. Chem., 44, 3650 (2006).

11. H. Wu, J. Xu, Y. Liu and P. Heiden, J. Appl. Polym. Sci., 72, 151 (1999).

12. R. Mezzenga and J.A.E. Mänson, J. Mater. Sci., 36, 4883 (2001).

13. L. Boogh, B. Pettersson, J.-A.E. Månson, Polymer, 40, 2249 (1999). 